\title{
LOW-RATE REDUCED COMPLEXITY IMAGE COMPRESSION USING DIRECTIONLETS
}

\author{
Vladan Velisavljevic ${ }^{1}$, Baltasar Beferull-Lozano ${ }^{2}$, Martin Vetterli $^{3,4}$, Pier Luigi Dragotti ${ }^{5}$ \\ ${ }^{1}$ Deutsche Telekom Laboratories, Germany, ${ }^{2}$ Universidad de Valencia, Spain, \\ ${ }^{3} \mathrm{EPFL}$, Switzerland, ${ }^{4}$ University of California, Berkeley, CA, ${ }^{5}$ Imperial College, UK
}

\begin{abstract}
The standard separable two-dimensional (2-D) wavelet transform (WT) has recently achieved a great success in image processing because it provides a sparse representation of smooth images. However, it fails to capture efficiently one-dimensional (1-D) discontinuities, like edges and contours, that are anisotropic and characterized by geometrical regularity along different directions. In our previous work, we proposed a construction of critically sampled perfect reconstruction anisotropic transform with directional vanishing moments (DVM) imposed in the corresponding basis functions, called directionlets. Here, we show that the computational complexity of our transform is comparable to the complexity of the standard 2-D WT and substantially lower than the complexity of other similar approaches. We also present a zerotree-based image compression algorithm using directionlets that strongly outperforms the corresponding method based on the standard wavelets at low bit rates.
\end{abstract}

Index Terms - Image coding, Image orientation analysis, Image segmentation, Wavelet transforms

\section{INTRODUCTION}

Providing efficient transform-based representations of images is an important problem in many areas of image processing, like approximation and compression. An efficient representation requires sparsity, that is, most of information has to be contained in a few largemagnitude coefficients.

The standard 2-D WT has become very successful because it provides a sparse multiresolution representation of smooth images owing to vanishing moments in the high-pass filters (or zeros at $\omega=0$ ) [1]. It also has a low computational complexity and simple filter design because of the separable filtering and subsampling operations.

However, the performance of the 2-D WT is limited by the $s p a-$ tial isotropy and two-directional construction of the corresponding basis functions, that is, the filtering and subsampling operations are applied symmetrically across scales only along the horizontal and vertical directions. For that reason, the standard 2-D WT fails to provide a sparse representation of 1-D discontinuities (edges and contours) in images. These features are characterized by a geometrical coherence that is not properly captured by the isotropic wavelet basis functions. Thus, to provide an efficient representation of edges and contours, the basis functions are required to be anisotropic and to have DVM along more than the two standard directions. Several

This work was supported by the Swiss National Foundation, Grant No. 200020-103729 and, in part, by EPSRC, Grant No. GR/S57631/01. previous approaches, like curvelets [2], contourlets [3], bandelets $[4,5]$ and wedgeprints [6], have already addressed this difficult task. However, these methods have higher complexity than the standard 2-D WT and require non-separable filtering and filter design. Furthermore, these transforms are often oversampled, thus, making it non-trivial to have efficient image compression methods.

In our previous work [7, 8, 9], we designed critically sampled anisotropic basis functions with DVM in any two directions with rational slopes, which we called directionlets. Our basis construction retains the separable processing and the computational simplicity of the standard 2-D WT. We showed that directionlets outperformed the standard 2-D WT in non-linear approximation (NLA) of images without a substantial increase of complexity. In [9], we also analyzed the approximation power of directionlets when applied to smooth synthetic images with one discontinuity curve.

In this paper, we analyze computational complexity of our transform and compare it to the complexity of other similar transforms. We also focus on image compression at low bit rates using directionlets and we present the results of compression of natural images. We propose a compression method based on multiscale wavelet structures, called zerotrees (originally introduced in [10]). Here, we adapt the zerotrees to directionlets and we call the resulting construction directional zerotrees. Furthermore, we show that the new image coder significantly outperforms the coder explained in [10] at low bit rates with the same order of computational complexity of the implemented transform.

The outline of the paper is as follows. In Section 2, we review the construction of directionlets and the asymptotic approximation behavior for smooth synthetic images. In Section 3, we analyze the computational complexity of our transform and compare it to the complexity of several similar transforms. Then, we present the coder based on directionlets and results on compression of natural images in Section 4. Finally, we conclude in Section 5.

\section{REVIEW OF DIRECTIONLETS}

The construction of directionlets has been explained in detail in our previous work $[7,8,9]$. Here, we only revisit the basic ideas.

As shown in [7], directionlets are constructed as basis functions of the so-called skewed anisotropic wavelet transforms (S-AWT). These transforms provide anisotropy of the basis functions and impose DVM along any two directions with rational slopes ${ }^{1}$ [7]. At the

\footnotetext{
${ }^{1}$ Recall that an $L$ th order DVM along the direction with a rational slope $r=b / a$ is equivalent to requiring the $z$-transform of a basis function to have a factor $\left(1-z_{1}^{-a} z_{2}^{-b}\right)^{L}$.
} 


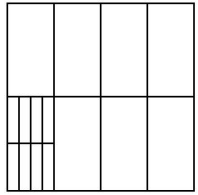

(a)

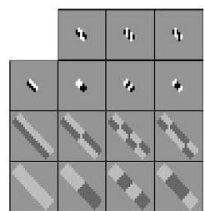

(b)

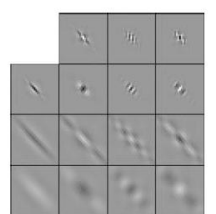

(c)
Fig. 1. The S-AWT allows for an anisotropic iteration of the filtering and subsampling operations applied along two different directions. (a) The decomposition in frequency for two iterations. The basis functions obtained from the (b) Haar and (c) biorthogonal "9-7" 1-D scaling and wavelet functions.

same time and very importantly, this method retains the 1-D filtering and subsampling operations, in addition to the simplicity of processing and filter design of the standard 2-D WT. Furthermore, the basis functions are critically sampled while ensuring perfect reconstruction.

Two examples of directionlets are shown in Fig. 1(b) and (c). These basis functions are constructed using the frequency decomposition illustrated in Fig. 1(a) and the Haar and biorthogonal "9-7" 1-D filter-banks, respectively.

Directionlets have been shown [7,8,9] to improve substantially the efficiency of representation of images that contain anisotropic structures in different orientations. They outperform the theoretical approximation power of the standard 2-D WT, as reviewed next.

\subsection{Non-linear Approximation of Images}

The task of image approximation is to represent an image by a subset of retained transform coefficients, while the rest of them is set to zero. In NLA, the indexes of the retained coefficients are adapted to the content of the signal.

The quality of the approximation is commonly measured in terms of mean-square error (MSE), that is, for a signal $\mathrm{x}$ and its approximation using $N$ coefficients $\hat{\mathbf{x}}_{N}$, the MSE is given by $\left\|\mathbf{x}-\hat{\mathbf{x}}_{N}\right\|^{2}$. The asymptotic rate of decay of the MSE, as $N$ tends to infinity, is a fundamental approximation property of the transform and this rate allows us to compare approximation performance of different transforms.

Mallat [11] showed that, for the $C^{2} / C^{2}$ class of images, ${ }^{2}$ the best decay of the MSE that can be achieved is $O\left(N^{-2}\right)$. The standard 2-D WT, instead, can achieve only a rate $O\left(N^{-1}\right)$ and is, therefore, suboptimal. Methods, like bandelets [4, 5] and wedgeprints [6] achieve optimal performance. Other methods, like curvelets [2] and contourlets [3] that achieve $O\left(N^{-2}(\log N)^{3}\right)$ are nearly optimal. However, as we show in Section 3, the complexity of all these algorithms is substantially higher than the standard 2-D WT and also directionlets.

Recall that natural images have geometrical features that vary over space. Thus, directionality is considered as a local characteristic, defined in a small neighborhood. Since directionlets are constrained to have DVM along only two directions, this implies the need for using spatial segmentation as a way of partitioning an image into smaller segments with one or a few dominant directions per segment. In our method, we use the quadtree segmentation (the

\footnotetext{
${ }^{2}$ Images that consist of two $C^{2}$ smooth regions separated by a $C^{2}$ smooth contour.
}

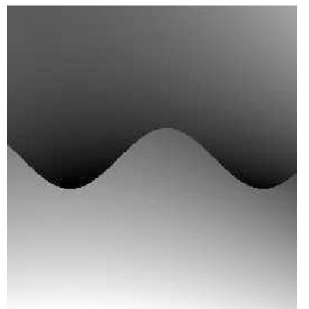

(a)

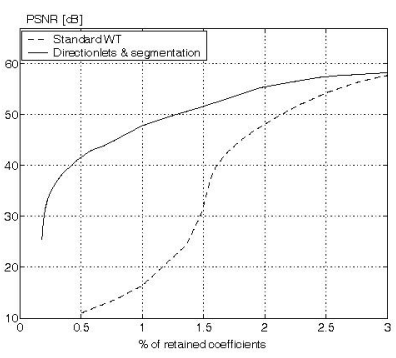

(b)
Fig. 2. An example of NLA of an image from the class $C^{2} / C^{2}$. (a) An image from the class $C^{2} / C^{2}$ is approximated using the standard WT and the S-AWT with spatial quadtree segmentation. (b) The MSE expressed in terms of PSNR is significantly reduced in the case when the S-AWT is applied.

whole image or each segment is divided into four equal segments), as the simplest segmentation method. Then, in each spatial segment, the corresponding directionlets are constructed using different directions adapted to the content of each segment.

It has been shown in $[7,8,9]$ that, by using spatial segmentation, directionlets can achieve a rate of decay of MSE equal to $O\left(N^{-1.55}\right)$, outperforming the standard 2-D WT. Fig. 2 shows an example of a $C^{2} / C^{2}$ image and gives a comparison of performance in NLA using directionlets and the 2-D WT. Notice that the shown graph compares the NLA performance only at lower rates because most of important coefficients are captured by a small portion of directionlets.

We emphasize that, although the rate of decay of the MSE achieved in our method is slower than the theoretically optimal rate, directionlets retain (a) low computational complexity and (b) critical sampling.

In the next section, we analyze the computational complexity of the applied S-AWT and compare it to the computational complexity of the other transforms that provide better approximation behaviors (contourlets, bandelets and wedgeprints). In Section 4, we show how the property of critical sampling allows for an efficient image compression method using directionlets based on Lagrangian optimization.

\section{COMPUTATIONAL COMPLEXITY OF DIRECTIONLETS}

Here, we calculate the order of the number of multiplications and additions required to implement the S-AWT. Then, we compare the result to the computational orders of the other methods.

As shown in Fig. 1(a) and in [7, 8], the S-AWT consists of iterated filtering and subsampling operations. Assuming that the subsampling operations do not carry any computational cost, each filtering operation is performed in $O(N \cdot L)$ multiplications and $O(N \cdot L)$ additions, where $N$ is the number of input samples and $L$ is the length of the applied filter. In the following theorem, we provide the precise order of computational complexity of the S-AWT.

Theorem 1 Given an $N \times N$ image, the number of operations required by the $S-A W T$ with $n_{1}$ and $n_{2}$ transform steps in the two transform directions, respectively, is of the order $O\left(L N^{2}\right)$, where $L$ is the length of the filter used in the transform. 
Table 1. Comparison of computational complexity of the 4 transforms for an $N \times N$ image.

\begin{tabular}{c|c}
\hline Directionlets & $O\left(L N^{2}\right)$ \\
\hline Contourlets & $O\left(L_{1} \cdot L_{2} N^{2}\right)$ \\
\hline Wedgeprints & $O\left(N^{2} \log _{2} N\right)$ \\
\hline Bandelets & $O\left(N^{2}\left(\log _{2} N\right)^{2}\right)$ \\
\hline
\end{tabular}

Proof: In one iteration block of the transform, there are

$$
O\left(\sum_{j=0}^{n_{1}+n_{2}-1} 2^{j} \cdot L N^{2} / 2^{j}\right)=O\left(\left(n_{1}+n_{2}\right) \cdot L N^{2}\right)
$$

multiplications and additions. Assume that the transform is iterated $J$ times. Then, the total number of operations is given by

$$
\begin{gathered}
O\left(2 \cdot \sum_{j=0}^{J-1}\left(n_{1}+n_{2}\right) L N^{2} \cdot 2^{-j\left(n_{1}+n_{2}\right)}\right)= \\
O\left(2 \cdot \frac{2^{n_{1}+n_{2}}}{2^{n_{1}+n_{2}}-1}\left(n_{1}+n_{2}\right) L N^{2}\left(1-2^{-J\left(n_{1}+n_{2}\right)}\right)\right),
\end{gathered}
$$

which results in $O\left(L N^{2}\right)$.

Notice that the computational complexity given by (1) is substantially lower than the complexity of the other similar methods. More precisely, bandelets require $O\left(N^{2}\left(\log _{2} N\right)^{2}\right)$ operations [4]. Wedgeprints implementation requires to perform a search in a large dictionary of linear edges and, thus, the complexity of processing grows rapidly with the size of image, as $O\left(N^{2} \log _{2} N\right)$ [6]. Furthermore, contourlets have complexity of the order $O\left(L_{1} \cdot L_{2} N^{2}\right)$, where the implemented filters are purely 2-D and have the size $L_{1} \times$ $L_{2}$. Therefore, the number of multiplications and additions is much higher (in the sense that the constant that is involved is higher) than in the case of directionlets [3]. Table 1 summarizes the comparison of computational complexity of these methods.

\section{COMPRESSION OF IMAGES}

Compression using orthogonal transform can be considered as an extension of NLA. It consists of approximation, indexing the retained coefficients, quantization and entropy coding.

Our goal in this section is to show that directionlets can improve substantially the performance of wavelet-based compression methods as compared to the standard 2-D WT. For that reason, we use the wavelet zerotree coder proposed in [10] in both cases. However, in our coder, we modify the multiscale zerotree structure of wavelet coefficients in such a way that the new parent-children relation among coefficients depends on anisotropy and orientation of directionlets (basis functions), as explained in the sequel. Notice that, although there exist newer and more efficient wavelet-based coders (like SPIHT [12] or SFQ [13]), we restrict ourselves to zerotrees for the sake of simplicity in the comparison. However, since similar zerotree-like relations among coefficients are exploited in these methods, their efficiency can also be improved using directionlets.

Recall that the standard zerotree structure is defined in such a way that each wavelet coefficient, except the ones at the finest scale, has a certain number of children, that is, the coefficients at the next

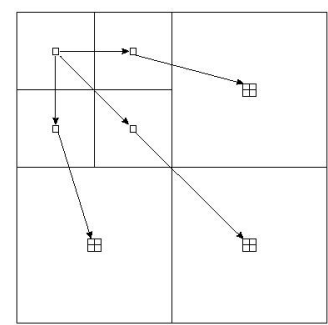

(a)

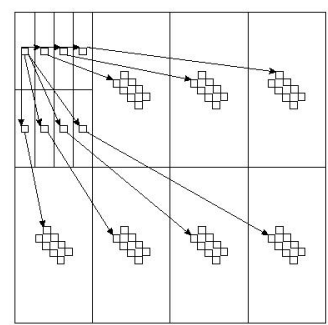

(b)
Fig. 3. The zerotrees of wavelet coefficients in the case of (a) the standard 2-D WT and (b) S-AWT along $+45^{\circ}$ and $-45^{\circ}$ (directional zerotrees).

finer scale that correspond to the same spatial location and orientation as the parent coefficient [10] (see Fig. 3(a)). The set of children is isotropic and aligned along the horizontal and vertical directions, following the properties of the standard 2-D WT. On the other hand, in the case of our transform, since directionlets are anisotropic and oriented in different directions, the corresponding children are grouped in anisotropic and oriented sets, as shown in Fig. 3(b). We call these structures directional zerotrees. The anisotropy and orientation of the sets of children is the same as that used in the construction of directionlets. Notice that directional zerotrees still retain the property of grouping coefficients across scales that belong to the same spatial location.

The adaptation of the coder to the content of image is performed in 3 optimization phases. In these phases, the optimal solution is found over (a) spatial quadtree segmentation, (b) choice of directions in each segment and (c) bit allocation for each chosen segment and directions. Since directionlets are critically sampled, we can use the Lagrangian optimization process easily in each optimization phase (note that this is not the case in other overcomplete methods).

The whole coding algorithm consists of 5 steps: $^{3}$

(i) The image is segmented in a full quadtree until a predetermined depth of spatial segmentation is reached.

(ii) In each segment, the S-AWT is performed for all possible pairs of directions from a predetermined set.

(iii) In each segment and for each pair of directions, the zerotree encoding algorithm is applied on the corresponding directionlets using directional zerotrees. The optimal encoding bit rate is chosen so that the minimal Lagrangian cost is achieved given a predetermined Lagrangian multiplicator.

(iv) In each segment, the optimal pair of directions is chosen so that the corresponding Lagrangian cost is minimal.

(v) In each segment, if the sum of Lagrangian costs of the children-segments is larger than the Lagrangian cost of the current segment, the children are pruned.

The improvement of the quality of compressed images, when compared to the results of the standard zerotree coding algorithm, is significant, especially at low bit rates. Fig. 4 shows the compression results of the synthetic $C^{2} / C^{2}$ image shown in Fig. 2(a) in the case of the standard and the directional method. The PSNRs of the reconstructions are $26.33 \mathrm{~dB}$ and $38.62 \mathrm{~dB}$, respectively, where the operational bit rate is the same and equal to 0.0263 bpp. In Fig. 4(a),

\footnotetext{
${ }^{3}$ Due to lack of space, we provide only a brief explanation of the coding process. For more details, we refer to [8].
} 


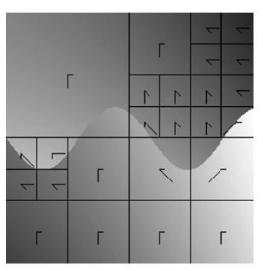

(a)

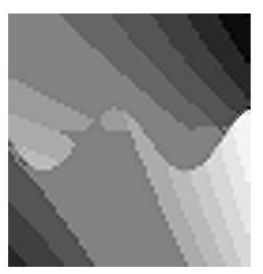

(b)

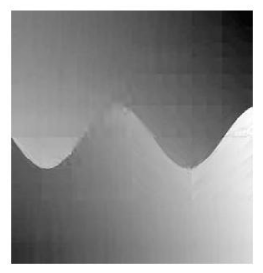

(c)
Fig. 4. The $C^{2} / C^{2}$ image shown in Fig. 2(a) is compressed using the zerotree-based method with the standard 2-D WT and the adaptation of the S-AWT to the content of each spatial segment. The depth of the transform decomposition in both cases is equal. (a) The optimal spatial segmentation and choice of directions. (b) The reconstruction obtained using the standard 2-D WT at the bit rate $0.0263 \mathrm{bpp}$ has the PSNR equal to $26.33 \mathrm{~dB}$. (c) The reconstruction obtained using directionlets at the same bit rate has the PSNR equal to $38.62 \mathrm{~dB}$.

the optimal spatial segmentation and choice of directions are shown. Fig. 5 presents a similar comparison between the performance of the two methods when applied to the natural test image Lena. The new method based on directionlets also outperforms the standard algorithm at low bit rates. The two reconstructions are made at the bit rate $0.0395 \mathrm{bpp}$ and the PSNRs are $23.51 \mathrm{~dB}$ and $26.07 \mathrm{~dB}$, respectively.

Notice that the optimization phases introduce a new computational complexity in the whole compression algorithm. This additional complexity is quadratic depending on the total number of possible directions and linear depending on the maximally allowed depth of spatial segmentation. However, in practice, we have observed that only 4 directions are enough to achieve a good performance. At the same time, good results are obtained with 3 levels of spatial segmentation. Thus, in practice, the increase in complexity is not substantial and affects only the constant. The order of the total complexity remains the same and is equal to $O\left(L N^{2}\right)$.

Similarly, the optimization process requires some overhead bits to encode the optimal solution. However, because of the small number of possibilities in practice, this additional bit rate is negligible (107 out of 6895 bits in the case shown in Fig. 4 and 118 out of 10355 bits for the example shown in Fig. 5) and does not affect the overall performance of the coder.

Notice also that spatial quadtree segmentation generates a blocking effect. However, the effect is not significant and does not affect the perceptual quality of the reconstructed image.

\section{CONCLUSION}

We analyzed the computational complexity of the anisotropic transform with DVM along different directions proposed in our previous work. The transform has the same order of complexity as the standard 2-D WT and substantially lower order when compared to other similar transforms. We also showed that the corresponding basis functions (directionlets) can be efficiently implemented in the zerotree-based image compression algorithm instead of the standard wavelets. The achieved results are significantly better than the results of the standard compression method, while retaining the same complexity.

\section{REFERENCES}

[1] M. Vetterli and J. Kovačević, Wavelets and Subband Coding. New Jersey, NJ: Prentice Hall PTR, 1995.

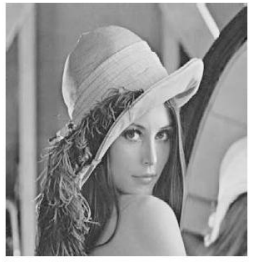

(a)

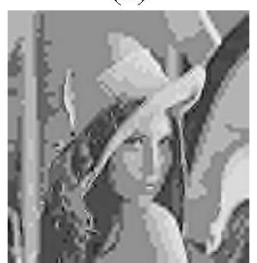

(c)

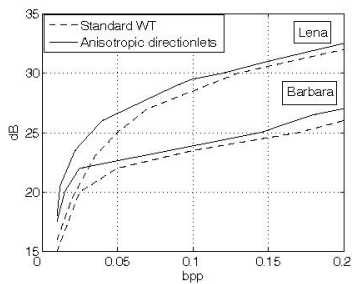

(b)

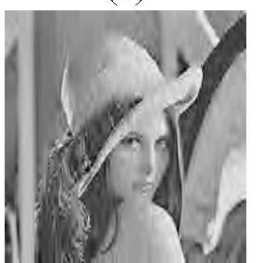

(d)
Fig. 5. (a) The original test image Lena. (b) The numerical comparison between the two compression methods at low bit rates for two test images: Lena and Barbara. The transforms in both methods have the same depth of decomposition. (c) The reconstruction obtained using the standard 2-D WT at the bit rate $0.0395 \mathrm{bpp}$ has the PSNR equal to $23.51 \mathrm{~dB}$. (d) The reconstruction obtained using directionlets at the same bit rate has the PSNR equal to $26.07 \mathrm{~dB}$.

[2] E. J. Candès and D. L. Donoho, "Curvelets - a surprisingly effective nonadaptive representation for objects with edges," in Curve and Surface Fitting, A. Cohen, C. Rabut, and L. L. Schumaker, Eds. Saint-Malo: Vanderbilt University Press, 1999.

[3] M. N. Do and M. Vetterli, "The contourlet transform: An efficient directional multiresolution image representation," IEEE Trans. Image Processing, vol. 14, pp. 2091-2106, Dec. 2005.

[4] E. L. Pennec and S. Mallat, "Sparse geometric image representations with bandelets," IEEE Trans. Image Processing, vol. 14, pp. 423-438, Apr. 2005.

[5] G. Peyré and S. Mallat, "Discrete bandelets with geometric orthogonal filters," in Proc. IEEE International Conference on Image Processing (ICIP2005), Genova Italy, Sept. 2005.

[6] M. Wakin, J. Romberg, H. Choi, and R. Baraniuk, "Wavelet-domain approximation and compression of piecewise smooth images" IEEE Trans. Image Processing, vol. 15, pp. 1071-1087, May 2006.

[7] V. Velisavljević, B. Beferull-Lozano, M. Vetterli, and P. L. Dragotti, "Directionlets: Anisotropic multi-directional representation with separable filtering," IEEE Trans. Image Processing, vol. 15, pp. 1916-1933, July 2006.

[8] V. Velisavljević, "Directionlets: anisotropic multi-directional representation with separable filtering." Ph.D. dissertation, School of Computer and Communication Sciences, EPFL, Lausanne, Switzerland, 2005.

[9] V. Velisavljević, B. Beferull-Lozano, M. Vetterli, and P. L. Dragotti, "Approximation power of directionlets," in Proc. IEEE International Conference on Image Processing (ICIP2005), Genova, Italy, Sept. 2005.

[10] J. M. Shapiro, "Embedded image coding using zerotrees of wavelet coefficients?" IEEE Trans. Signal Processing, vol. 41, pp. 3445-3463, Dec. 1993.

[11] S. Mallat, A Wavelet Tour of Signal Processing. San Diego, CA: Academic Press, 1997.

[12] A. Said and W. A. Pearlman, "A new, fast, and efficient image codec based on set partitioning in hierarchical trees," IEEE Trans. Circuits Syst. Video Technol., vol. 6, pp. 243-250, June 1996.

[13] Z. Xiong, K. Ramchandran, and M. T. Orchard, "Space-frequency quantization for wavelet image coding," IEEE Trans. Image Processing, vol. 6, pp. 677-693, May 1997. 